\title{
Modal Cutoff in Coaxial Transmission Lines of Conical and Cylindrical Geometries*
}

\author{
Claude M. Weil, Bill F. Riddle, David R. Novotny, and Robert T. Johnk \\ Radio-Frequency Technology Division, National Institute of Standards and Technology (NIST), \\ 325 Broadway, Boulder, CO 80305-3328, USA
}

\begin{abstract}
We present numerical data on normalized cutoff wavelength versus cone half-angle for the first three higherorder $\mathbf{T E}_{\mathrm{m} 1}$ modes, as well as four other modes of interest, in conical coaxial transmission lines (the "co-conical" line). These are given as a function of the ratio of outer-to-inner cone half-angles (proportional to line impedance) for outer cone half-angles of 10 and 22.5 degrees. Results were compared to those for the coaxial transmission line of cylindrical geometry and found to be qualitatively similar.
\end{abstract}

\section{INTRODUCTION}

The microwave applications for coaxial transmission lines of conical geometry have been somewhat limited. Currently, there is interest in using such lines (what we have termed a "co-conical line") for accurately calibrating broadband power-density and E-field probes at high incident field levels [1]. A prototype version of a one-meter long coconical system, being developed at NIST, is illustrated in Fig. 1. Because we needed to determine the usable TEMmode bandwidth of this structure and because such data appeared to be unavailable in the literature, we sought to generate these data ourselves.

The theory for waves guided within conical structures was first developed more than sixty years ago by Schelkunoff [2,3]. Both Schelkunoff and Marcuvitz [4] discuss the coconical line (Marcuvitz termed it a "Conical Waveguide"). Neither Schelkunoff or Marcuvitz attempted to derive any numerical data on modal cutoff in such structures, because very little tabulated data on Legendre functions of fractional order were then available.

In order to check the accuracy of the root-finding routines used in our mathematical software, we also derived modal cutoff data for higher-order modes in the coaxial (cylindrical) transmission line. We also wanted to qualitatively compare our results for the co-conical line

* Publication of the US Government, not subject to US Copyright.

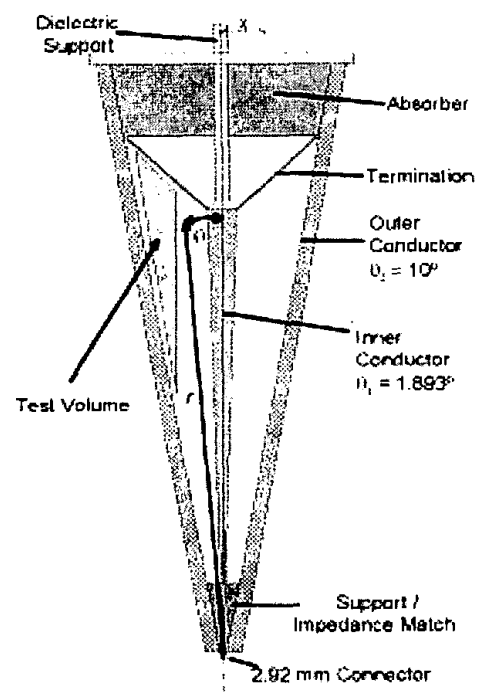

Fig. 1. Illustrated Diagram of the NIST Prototype Co-Conical Field Generation System.

with those for the cylindrical coaxial line. Although the latter problem has been considered by many workers [2]-[6] over the past sixty years, we were surprised to find that serious data limitations exist in the available literature on this problem, which significantly affect the usability of these data. Therefore, we have also included a comprehensive and much simpler data plot.

\section{THEORY}

Only the transcendental equations of Schelkunoff's theory [2,3], which define modal cutoff in coaxial conical and cylindrical lines, will be repeated here. For a TM spherical wave propagating in the region between two coaxial conical conductors with inner and outer half-angles $\bullet_{1}$ and $\bullet_{2}$ 
respectively, modal cut-off is defined by the following relationship:

$$
\frac{P_{x_{n, m} y^{\bullet m}}^{y^{*}}\left(\cos ^{\bullet}{ }_{1}\right)}{Q_{x_{n, m} m}^{y^{\bullet} m}\left(\cos ^{\bullet}\right)} \cdot \frac{P_{x_{n, m}}^{y^{\bullet} m}\left(\cos ^{\bullet}{ }_{2}\right)}{Q_{x_{n, m} m}^{y \bullet m}\left(\cos ^{\bullet}{ }_{2}\right)},
$$

where $P_{x}^{y}\left(\cos ^{\bullet}\right)$ and $\mathrm{Q}_{\mathrm{x}}^{\mathrm{y}}\left(\cos ^{\bullet}\right)$ respectively represent associated Legendre functions, of the first and second kind, with order $x$ and degree $y$. A similar relationship defines modal cutoff for all TE modes:

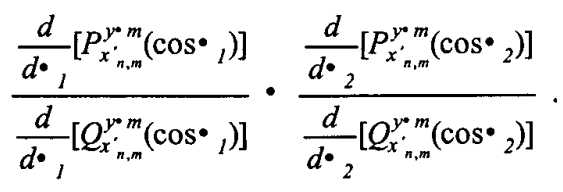

In the above equations, $y$ is an integer $m$, and $x_{n, m}$ and $x_{n, m}$ respectively represent the $n$th eigenfunction root (where $n$ is a nonzero integer) of $x$, degree $m$, that satisfies equations (1) and (2). It is straightforward to show that the normalized (dimensionless) cutoff wavelength for higherorder modes in the co-conical line is given by

$$
\left[\cdot{ }_{c}\right]_{T M_{m n}} / r \cdot 2 \cdot\left[x_{n, m}\left(x_{n, m} \cdot 1\right)\right]^{\cdot 1 / 2}
$$

for TM modes and

$$
\left[{ }_{c}\right]_{T E_{m n}} / r \cdot 2 \cdot\left[x_{n, m}^{\prime}\left(x_{n, m}^{\prime} \cdot 1\right)\right]^{1 / 2}
$$

for TE modes.

Similarly, for a TM plane wave propagating in the region between two coaxial cylindrical conductors with inner and outer radii $a_{1}$ and $a_{2}$ respectively, modal cut-off is defined by the following relationship:

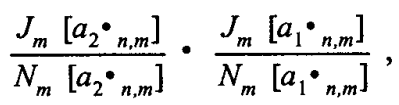

where $J_{m}\left[a^{\bullet}{ }_{n, m}\right]$ and $N_{m}\left[a^{\bullet}{ }_{n, m}\right]$ respectively represent Bessel and Neumann functions of order $y$. A similar expression is derived for the TE modes:

$$
\frac{\frac{d}{d a_{1}}\left(J_{m}\left[a_{2} \cdot{ }_{n, m}\right]\right)}{\frac{d}{d a_{1}}\left(N_{m}\left[a_{2} \cdot{ }_{n, m}\right]\right)} \cdot \frac{\frac{d}{d a_{2}}\left(J_{m}\left[a_{1} \cdot{ }_{n, m}\right]\right)}{\frac{d}{d a_{2}}\left(N_{m}\left[a_{1} \cdot{ }_{n, m}\right]\right)}
$$

In the above equations, ${ }_{n, m}$ and $\bullet_{n, m}$ respectively represent the $n$th nonzero root, integer order $m$, that satisfies equations (5) and (6). For the cylindrical geometry, the normalized wavelength is given by :

$$
\left(\cdot{ }_{c}\right)_{r M_{m n}} / a_{2} \cdot 2 \cdot / \cdot{ }_{n, m}
$$

for TM modes and

$$
\left(\cdot{ }_{c}\right)_{T E_{m n}} / a_{2} \cdot 2 \cdot 1 \cdot{ }_{n, m}
$$

for the TE modes.

\section{RESULTS}

Derivation of the numerical roots of equations (1), (2), (5), and (6) was performed using a commercially available mathematical software package. We found that this search routine generally worked well, provided that the root search was confined to a narrow range that needed to be specified in advance. Fig. 2 shows data on ${ }_{c} / r$ versus the ratio of outer-to-inner cone half-angles $\bullet_{2} / \bullet_{1}$ in the co-conical line for the first three higher-order $\mathrm{TE}_{\mathrm{ml}}$ modes, as well as the $\mathrm{TM}_{01}, \mathrm{TM}_{11}, \mathrm{TE}_{12}$, and $\mathrm{TM}_{02}$ modes. The values chosen for ${ }_{2}{ }^{\bullet}{ }_{1}$ above 1.1 correspond to transmission-line impedances $Z_{0}$ of $10,25,50,75,100$, and $125 \cdot$. The relation between ${ }_{2}$ $1 \bullet$, and $Z_{0}$ is given in $[2,3]$. Fig. 2 a gives results for an outer cone angle of ${ }_{2}=10^{\circ}$, while Fig. 2b shows data for ${ }_{2}=$ $22.5^{\circ}$.

Similarly, Fig. 3 shows data on ${ }_{c} / a_{2}$ versus the ratio of outer-to-inner radii $a_{2} / a_{1}$ in the coaxial cylindrical line for exactly the same set of higher-order modes as given in Fig. 2 and the same values of $Z_{0}$. The relation between $a_{2} / a_{1}$ and $Z_{0}$ is also given in [2,3]. We compared these data with available literature values, including the tabulated data of Marcuvitz [4] and graphical plots of Ramo, Whinnery and 
Van Duzer [5] and Harvey [6] for a number of test cases involving low-order TM and TE modes with values of $a_{2} / a_{1}$ $=1.2,2.3$, and 3.5. This was not a straight-forward process owing to the manner in which cutoff data are currently presented in the literature. Marcuvitz lists values of ${ }_{n, m}$ and - $_{n, m}$ for varying values of $a_{2} / a_{1}$ in three separate tables, depending on the mode series being considered. Additional computations are then needed in order to derive values of - ${ }_{c} / a_{2}$. In $[5,6]$, cutoff data are given in terms of an ordinate parameter that is some function of $\bullet{ }_{c} / a_{2}$, and plotted against either $a_{2} / a_{1}$ or $a_{1} / a_{2}$. This meant that corrections had to be applied to these data, before comparisons could be undertaken. Following these various corrections, our data were found to agree closely with Marcuvitz's data [4] and the plotted data $[5,6]$. We therefore concluded that our rootfinding software has given us valid data.

\section{DISCUSSION}

We see that the cutoff data shown in Figs. 2 and 3 are qualitatively, but not quantitatively, quite similar and that the order in which the higher-order modes appear is the same. Note that for both geometries, the normalized cutoff wavelength for the $T E_{m 1}$ modes approaches a finite asymptotic value as $\bullet_{2} / \bullet_{1}$ and $a_{2} / a_{1}$ approach unity. However, these cases have no physical meaning, despite the apparent availability of a mathematical solution.

The available data on cutoff in coaxial cylindrical lines have not been well presented in the literature. We believe that Fig. 3 presents these data over a wider range of $a_{2} / a_{1}$ and for more modes, in a manner that is much simpler and easier to interpret, and therefore more useable to designers.

\section{REFERENCES}

[1] D.R. Novotny, R.T. Johnk, A. Ondrejka: "Improved wideband Antenna Test Cell: the Co-Conical Field Generation System." Proceedings of AMTA Conference, pp. 144-149, Monterey, CA, 4-8 Oct. 1999

[2] S.A. Schelkunoff: "Transmission Theory of Spherical Waves," AIEE Transactions, Vol. 38, pp. 744-750, 1938.

[3] A. Schelkunoff: Electromagnetic Waves, D. Van Nostrand Co., Inc: Princeton, NJ, 1943.

[4] N. Marcuvitz (ed): "Waveguide Handbook ", Radiation Laboratory Series, Vol. 10, McGraw-Hill Co., Inc.: New York, NY, 1951.

[5] S. Ramo, J.R. Whinnery, and T. Van Duzer: "Eields and Waves in Communication Electronics", $2^{\text {nd }}$. Edition, John Wiley \& Sons, Inc.: New York, NY, 1984.

[6] A.F Harvey: "Microwave Engineering", Academic Press, Inc., London and New York, 1963.

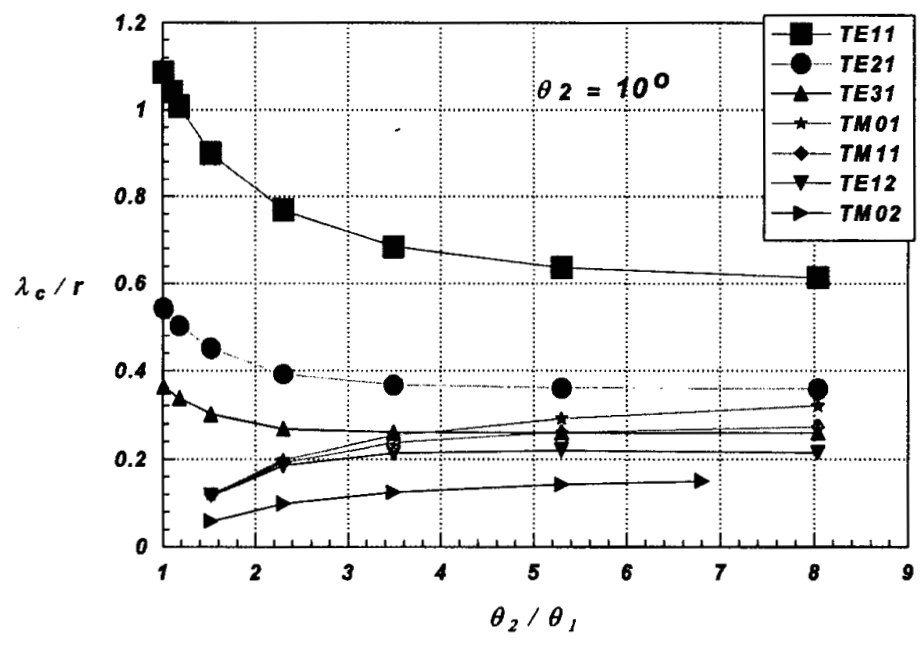

Fig. 2a. Normalized cutoff wavelength ${ }_{c} / r$ versus the ratio of outer-to-inner cone half-angles $\bullet_{2} /{ }_{1}$ for the first three higherorder $\mathrm{TE}_{\mathrm{m} 1}$ modes in the co-conical line, as well as other modes of interest; $\bullet_{2}=10^{\circ}$. 


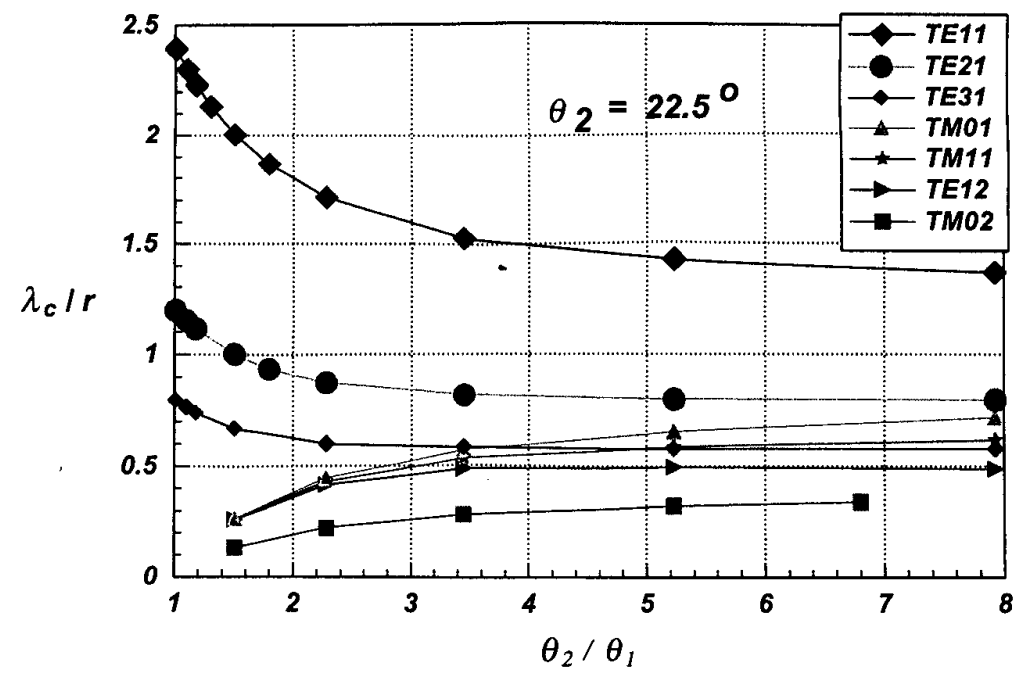

Fig. 2b. Normalized cutoff wavelength ${ }_{c}{ }_{c} r$ versus the ratio of outer-to-inner cone half-angles $\bullet f^{\bullet}$, for the first three higherorder $\mathrm{TE}_{\mathrm{m} l}$ modes in the co-conical line, as well as other modes of interest; $\bullet_{2}=22.5^{\circ}$.

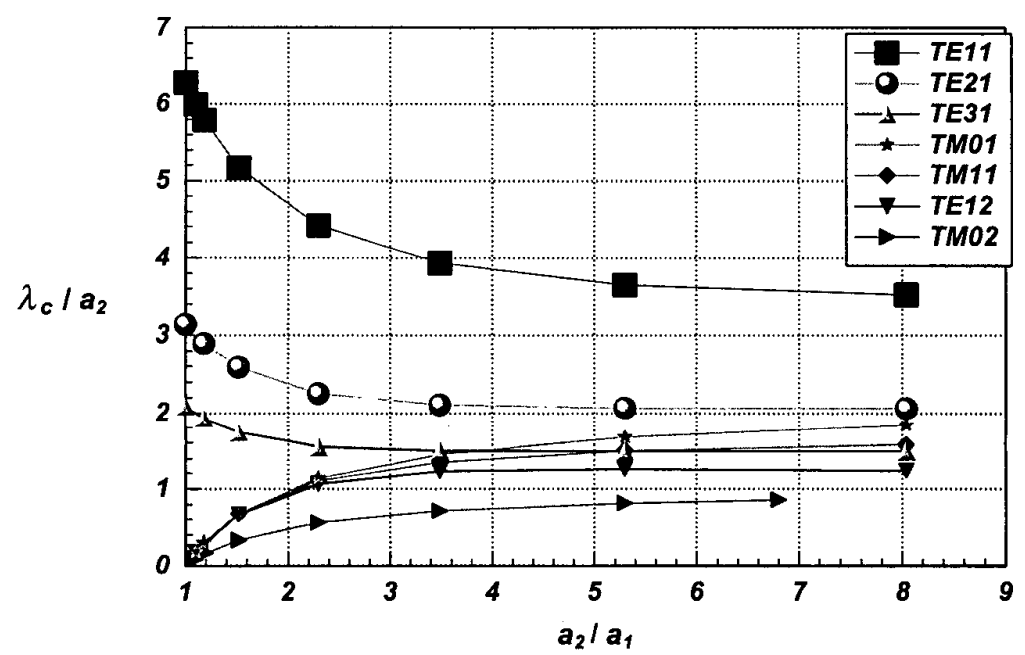

Fig. 3. Normalized cutoff wavelength ${ }_{c} / a_{2}$ versus the ratio of outer-to-inner radii $a_{2} / a_{1}$ for the first three higher-order $\mathrm{TE}_{\mathrm{ml}}$ modes in the coaxial transmission line, as well as various other modes of interest. 